\title{
Molecular evidence of bird-eating behavior in Nyctalus aviator
}

\author{
Olga Heim $^{1,2}$ (D) Anna I. E. Puisto ${ }^{2} \cdot$ Dai Fukui $^{3} \cdot$ Eero J. Vesterinen ${ }^{2,4}$
}

Received: 8 November 2018 /Revised: 15 May 2019 / Accepted: 30 May 2019/Published online: 22 June 2019

(C) The Author(s) 2019

\begin{abstract}
Insectivorous bats consume a large variety of food items. Previous observations of feathers found in feces led to the hypothesis that the birdlike noctule (Nyctalus aviator, Vespertilionidae) could prey on birds. To test the hypothesis, we analyzed fecal samples from six species (Barbastella pacifica, Murina hilgendorfi, Myotis frater, N. aviator, Plecotus sacrimontis, and Vespertilio sinensis) collected from central Hokkaido, Japan, via DNA barcoding. We identified the presence of the Middendorff's grasshopper warbler (Locustella ochotensis) in the diet of a pregnant individual of $N$. aviator. All the other samples proved negative regarding bird prey DNA. This is the first time that the consumption of a bird by N. aviator is confirmed with molecular evidence. Our findings add invaluable insight into the diet of this bat and its potentially opportunistic foraging behavior.
\end{abstract}

Keywords Nyctalus aviator $\cdot$ Locustella ochotensis $\cdot$ Bird prey $\cdot$ DNA barcoding $\cdot$ Sanger sequencing

\section{Introduction}

There are about 1300 species of bats worldwide, of which the vast majority are insectivorous (Fenton and Simmons 2015; Vesterinen et al. 2013; Vesterinen et al. 2016; Vesterinen et al. 2018). Although invertebrate prey is usually widely available, many insectivorous bats are known to occasionally forage on other, rarer prey, including fish or birds (e.g., Aizpurua et al. 2013; Fukui et al. 2013; Ibáñez et al. 2016, Ma et al. 2003). While the frequency and significance of this behavior remain unstudied, it may serve as an important nutritional strategy

Electronic supplementary material The online version of this article (https://doi.org/10.1007/s10211-019-00319-5) contains supplementary material, which is available to authorized users.

Olga Heim

bats@o-heim.de

Eero J. Vesterinen

ejvest@utu.fi

1 Animal Ecology, University of Potsdam, D-14469 Potsdam, Germany

2 Biodiversity Unit, University of Turku, FI-20014 Turku, Finland

3 The University of Tokyo Hokkaido Forest, The University of Tokyo, Furano 079-1563, Japan

4 Department of Ecology, Swedish University of Agricultural Sciences, 75007 Uppsala, Sweden during key life stages, such as reproduction (see PopaLisseanu et al. 2007).

In Japan, Fukui et al. (2013) reported the occasional consumption of birds by $N$. aviator, but the prey species were not identified. Here, we use molecular tools to detect the presence of a bird in the diet of $N$. aviator and to confirm the prey species.

\section{Material and methods}

Samples were obtained from 2015 to 2017 within the framework of a bigger study on the diet of Japanese bats (unpublished data). All the trapping and DNA extraction methods were carried out in the same way for all the samples as explained below.

\section{Study area}

The undulating landscape of the study region in central Hokkaido, Japan, is characterized by a mixture of arable land and urban areas in the valleys, while mixed forests cover the hills (Fig. 1). The study was conducted during the summer months (May to September) of 2015-2017. In the humid continental climate, average temperatures range from 9 to $17{ }^{\circ} \mathrm{C}$ in May, which is the coolest month of the study period, and from 19 to $26^{\circ} \mathrm{C}$ in August, which is the warmest month of the 
year (Japan Meteorological Agency 2018). The mean precipitation from May to September ranges between 50 and 150 mm (Japan Meteorological Agency 2018).

\section{Bat trapping and fecal sampling}

Trapping sites were located in multiple locations in Hokkaido. Samples for Barbastella pacifica Kruskop, Kawai \& Tiunov, 2019, Murina hilgendorfi Peters, 1880, Myotis frater G.M. Allen, 1923, Plecotus sacrimontis Allen, 1908, and Vespertilio sinensis Peters, 1880 were collected between 2015 and 2017 using harp traps or mist nets during nighttime. Nyctalus aviator Thomas, 1911, was captured from a bat box during daytime in 2017 (Fig. 1). Basic morphometric measurements were recorded for all bats. Bat feces were collected, either directly from the individual while handling, or from the cotton bags used to hold the bats while processing, resulting in one to ten fecal pellets per individual (Table 1). Fecal pellets were stored in $70 \%$ ethanol at $-20^{\circ} \mathrm{C}$ until laboratory analysis.

\section{Laboratory work and data analysis}

DNA was extracted using QIAamp PowerFecal DNA Kit (Qiagen(/MoBio) cat. nr 12830-50, Qiagen, Hilden, Germany) following the manual (MoBio "Protocol: Detailed"; version 12192013) with following modifications (numbers refer to the step numbers in the manual). In step 1, we used whole fecal droppings as starting material (samples were dried briefly on clean paper). In step 6, we used a Tissue Lyser II (Cat No. 85300, Qiagen, Hilden, Germany) $2 \times 1 \mathrm{~min}$ at full speed. In step 14, we transferred $700 \mu 1$ of the supernatant into a clean collection tube and added $1125 \mu$ lof solution $\mathrm{C} 4$ in step 15. DNA was eluted into $100 \mu \mathrm{L}$ of C6 buffer as recommended in the protocol and stored at $-20{ }^{\circ} \mathrm{C}$ until subsequent analysis.

Fig. 1 The main map depicts the trapping site located north of Biei, while the inset map provides an overview of the site within Hokkaido.

Source: Main map: based on National Land numerical information: Land Use Fragmented Mesh Data "L03-b-14_6542", year 2014, provided by the Ministry of Land, Infrastructure, Transport and Tourism; Inset map: based on a map provided by the Geospatial Information Authority of Japan, http://www.gsi.go.jp/kankyochiri/ gm_japan_e.html, accessed: 6th of September 2018
We used a single bat-specific primer pair targeting the DNA barcode region of the mitochondrial cytochrome oxidase subunit I (COI; Walker et al. 2016) to confirm the presence of bird DNA in the bat feces. The PCR protocol followed Kaunisto et al. (2017), except that we used the MyTaq HS Red Mix (product nr BIO-25048, Bioline, UK). All the blank control reactions proved negative, that is, free of contamination. Successful PCR products were purified using A'SAP clean kit (product nr 80350, ArcticZymes, Trømssa, Norway) and sequenced by Sanger sequencing ( $5^{\prime}$ direction) at Macrogen Europe (Macrogen Inc., Seoul, South Korea). Sequences were trimmed for poor quality regions and primers were removed as described in Sorvari et al. (2012) using Geneious R6 (Kearse et al. 2012). Trimmed sequences were identified using BOLD systems (Ratnasingham and Hebert 2007) and blasted against the GenBank database (Altschul et al. 1990). As one of the sequences from $N$. aviator matched bird sequences in BOLD (Locustella ochotensis, $100 \%$ similarity), we re-analyzed the DNA from the fecal samples of the larger bats (forearm length over $40 \mathrm{~mm} ; n=25$; Table 1) because we assumed that they are more likely to prey upon birds. For this re-analysis, we used three bird-specific primer pairs targeting mitochondrial cytochrome oxidase subunit I and cytochrome b genes (PastorBeviá et al. 2014; Online Resource 1: Table S1). Successful reactions were purified using A'SAP clean kit and sequenced by Sanger sequencing ( $5^{\prime}$ direction) at Macrogen Europe. Even when the amplification of short CytB products could not be confirmed in the gels, we purified the PCR products and sequenced them. The resulting sequences were trimmed and identified as described above. To further confirm the bird species, we downloaded trace files for the three closest matches for COI sequence from BOLD (species and ProcessID for each record: Locustella ochotensis, KFIP03107; L. pleskei, KBBI031-07; and L. certhiola, KBPBR487-07) and four closest matches from GenBank for CytB-short

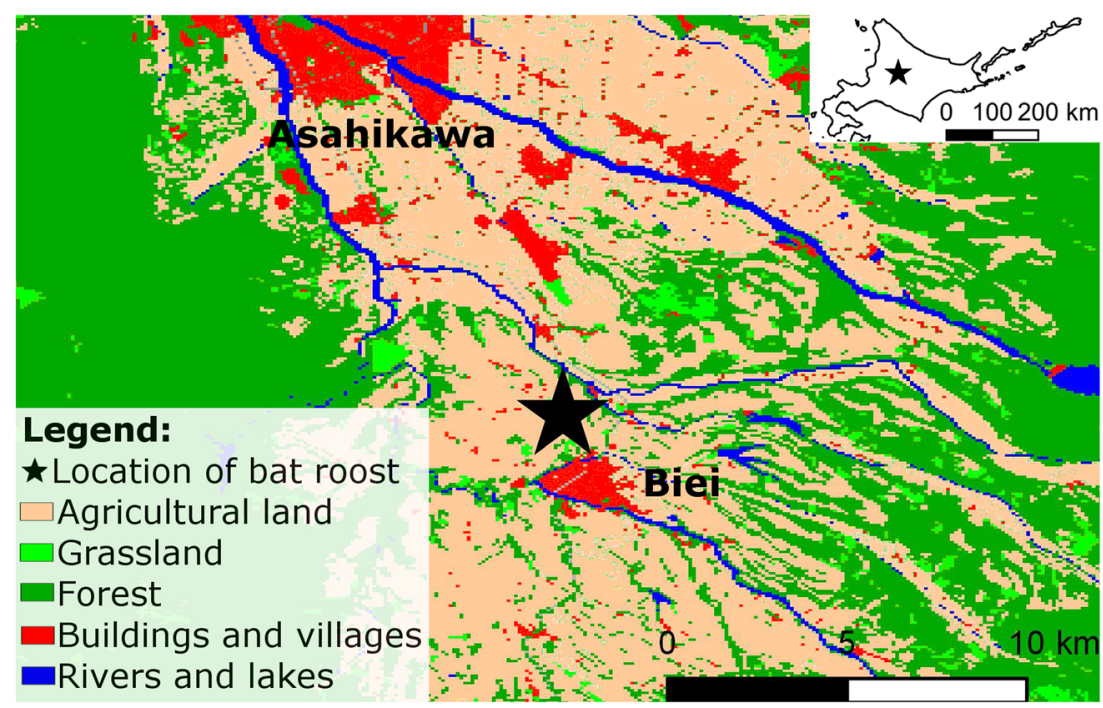


(accession numbers L. ochotensis, HQ706157; L. pleskei, AB261530; L. certhiola, HQ706154; and Megalurus pryeri, AB261528) and CytB-long (L. ochotensis, HQ706156; L. pleskei, AB261532; L. certhiola, HQ608848; and M. pryeri, AB261528). We then aligned our trimmed sequences with each corresponding reference set to see the actual nucleotide-level similarity. Detailed methods and alignments are available in Online Resource 1.

\section{Results}

The presence of bird DNA (Passeriformes, Sylvidae, L. ochotensis) in one sample of a female $N$. aviator was confirmed with the three primer pairs (COI, CytB-short, and CytB-long). The rest of the analyzed samples did not show evidence of bird DNA. We assigned the sequences from the one successful sample to a single bird species with $100 \%$ similarity. The sequences produced in this study are stored in GenBank with accession numbers MK900675 (COI), MK900676 (CytB-short), and MK900677 (CytB-long).

\section{Discussion}

Earlier evidence of predation of passerine birds by $N$. aviator was provided by Fukui et al. (2013), who sampled maternity colonies on Hokkaido and found bird feathers in fecal pellets from mid-May to June and September to late December. In this study, we found DNA of $L$. ochotensis in the feces of one pregnant $N$. aviator.

Table 1 Bat species, sampling year, sex, age, and number of samples (with one to ten fecal pellets per sample) analyzed in this study for each group

\begin{tabular}{llllll}
\hline Genus & Species & Year & Sex & Age & $n$ \\
\hline Barbastella & pacifica & 2015 & M & ad & 2 \\
Murina & hilgendorfi & 2015 & M & ad & 5 \\
Plecotus & sacrimontis & 2015 & M & ad & 3 \\
Vespertilio & sinensis & 2015 & $\mathrm{~F}$ & j & 1 \\
Murina & hilgendorfi & 2016 & $\mathrm{~F}$ & ad & 1 \\
Murina & hilgendorfi & 2016 & $\mathrm{M}$ & $\mathrm{NA}$ & 1 \\
Murina & hilgendorfi & 2017 & $\mathrm{M}$ & ad & 2 \\
Myotis & frater & 2017 & $\mathrm{M}$ & ad & 1 \\
Nyctalus & aviator & 2017 & $\mathrm{~F}$ & ad & 7 \\
Plecotus & sacrimontis & 2017 & $\mathrm{~F}$ & ad & 1 \\
Vespertilio & sinensis & 2017 & $\mathrm{~F}$ & $\mathrm{j}$ & 1 \\
\hline
\end{tabular}

Abbreviations: $\mathrm{M}=$ male, $\mathrm{F}=$ female, $\mathrm{ad}=$ adult $\mathrm{j}=$ juvenile, $\mathrm{NA}=$ not available
With a size range of $13.5-14.5 \mathrm{~cm}$ and a weight range of 19-23 g, L. ochotensis (Brazil 2009) is of similar size, but about half the body weight of $N$. aviator (Ohdachi et al. 2015). Similarly, $N$. lasiopterus has been reported to feed on Erithacus rubecula, Parus caeruleus and other migratory passerines in Italy and Spain during autumn (Dondini and Vergari 2000; Ibáñez et al. 2001; Popa-Lisseanu et al. 2007). These birds are about half the size of $N$. lasiopterus and highlight the ability of these bats to capture prey that is half their weight while flying (Ibáñez et al. 2016).

Locustella ochotensis is a migratory passerine that passes through the Aomori prefecture south of Hokkaido between early-June and late-June and arrives a few days later at its breeding grounds in Hokkaido (Ishizawa 1960). Since the breeding period of these birds starts with the arrival of females in June (Nagata 1986) and the sampling of $N$. aviator took place in early June, $N$. aviator was feeding on L. ochotensis between the end of the bird's migration period and beginning of its breeding period. Nyctalus lasiopterus and Ia io have been reported to feed on passerine birds largely during the migratory periods, when bats are thought to capture their prey at high altitudes (Ibáñez et al. 2016). These bat species, as well as $N$. aviator, are adapted to foraging in open space, with long and narrow wings that allow for fast flight and echolocation calls at low frequencies (Fukui et al. 2004) to detect objects at a large distance (Norberg and Rayner 1987). As L. ochotensis may fly at high altitudes at night during migration (Ishizawa 1960), it is probable that $N$. aviator also catches these birds by aerial-hawking.

In light of previous reports about bird predation during periods of bird migration, it appears unlikely that $N$. aviator would catch these warblers during their breeding period, as the warblers exhibit nocturnal flight activity only during migration (Ishizawa 1960).

Although our finding confirms the hypothesis that $N$. aviator feeds on passerine birds, this study is based on a single positive sample, which highlights the need for further, more extensive, and specific diet studies.

As Japan lies within the East Asian flyway - a migratory route for many species of passerines (Brazil 2009) — we hypothesize that $N$. aviator forages on other passerine bird species in order to fulfill similar dietary requirements as $N$. lasiopterus. In contrast, $N$. aviator may opportunistically feed on passerine birds as they represent a rich food source that is available only during a short seasonal period. To test these hypotheses, more studies are necessary.

Acknowledgments We thank Dr. Fujioka, Mr. Ujino, Mr. Hamai and Mr. Nakai for helping with the fieldwork. We wish to thank both the Biodiversity Unit and the Department of Biology at the University of Turku for allowing the use of molecular laboratories. BEng. Satu Mäkelä is thanked for her help in the laboratory. We acknowledge the CSC-IT Center for Science Ltd., Espoo, Finland, for the allocation of computational resources. Finally, we like to thank the reviewers and Dr. 
David A. Hill for their highly valuable, productive, and helpful comments and suggestions that contributed to the improvement of this manuscript. Funding information Open access funding provided by University of Turku (UTU) including Turku University Central Hospital. The study was financially supported by JSPS KAKENHI (Grant-in-aid for Scientific Research on Innovative Areas: Nos JP16H06542 and JP16K21735, Grant-in-aid for Scientific Research (C): No JP16K00568, DF), the Jane and Aatos Erkko Foundation, and Emil Aaltonen Foundation (EJV, AIEP). This study was supported by the Finnish Functional Genomics Centre, University of Turku and Åbo Akademi and Biocenter Finland.

\section{Compliance with ethical standards}

The authors declare that they have no conflict of interest.

Ethical approval The capture survey was conducted under the permission from the Ministry of Environment (Nos. 21-27-0077, 21-28-0087, and 21-29-0131) and Hokkaido Prefecture (Nos. 26-35 and 57), Japan. All applicable international, national, and/or institutional guidelines for the care and use of animals were followed.

Open Access This article is distributed under the terms of the Creative Commons Attribution 4.0 International License (http:// creativecommons.org/licenses/by/4.0/), which permits unrestricted use, distribution, and reproduction in any medium, provided you give appropriate credit to the original author(s) and the source, provide a link to the Creative Commons license, and indicate if changes were made.

\section{References}

Aizpurua O, Garin I, Alberdi A, Salsamendi E, Baagøe H, Aihartza J (2013) Fishing long-fingered bats (Myotis capaccinii) prey regularly upon exotic fish. PLoS One 8(11):e80163

Altschul SF, Gish W, Miller W, Myers EW, Lipman DJ (1990) Basic local alignment search tool. J Mol Biol 215(3):403-410

Brazil M (2009) Birds of East Asia: China, Taiwan, Korea, Japan, and Russia. A\&C Black

Dondini G, Vergari S (2000) Carnivory in the greater noctule bat (Nyctalus lasiopterus) in Italy. J Zool 251(2):233-236

Fenton MB, Simmons NB (2015) Bats: a world of science and mystery. University of Chicago Press

Fukui D, Agetsuma N, Hill DA (2004) Acoustic identification of eight species of bat (Mammalia: Chiroptera) inhabiting forests of southern Hokkaido, Japan: potential for conservation monitoring. Zool Sci 21(9):947-955

Fukui D, Dewa H, Katsuta S, Sato A (2013) Bird predation by the birdlike noctule in Japan. J Mammal 94(3):657-661

Ibáñez C, Juste J, García-Mudarra JL, Agirre-Mendi PT (2001) Bat predation on nocturnally migrating birds. PNAS 98(17):9700-9702

Ibáñez C, Popa-Lisseanu AG, Pastor-Beviá D, García-Mudarra JL, Juste J (2016) Concealed by darkness: interactions between predatory bats and nocturnally migrating songbirds illuminated by DNA sequencing. Mol Ecol 25(20):5254-5263

Ishizawa J (1960) On the distribution and migration of Locustella o. ochotensis. JJO 15(75):214-226
Japan Meteorological Agency (2018) Climate of Hokkaido district. Japan Meteorological Agency, Available from http://www.data.jma.go.jp/ $\mathrm{gmd} / \mathrm{cpd} /$ longfcst/en/tourist/file/Hokkaido.html, accessed: 14.05 . 2018)

Kaunisto KM, Roslin T, Sääksjärvi IE, Vesterinen EJ (2017) Pellets of proof: first glimpse of the dietary composition of adult odonates as revealed by metabarcoding of feces. Ecol Evol 7(20):8588-8598

Kearse M, Moir R, Wilson A, Stones-Havas S, Cheung M, Sturrock S, Buxton S, Cooper A, Markowitz S, Duran C, Thierer T, Ashton B, Meintjes P, Drummond A (2012) Geneious basic: an integrated and extendable desktop software platform for the organization and analysis of sequence data. Bioinformatics 28(12):1647-1649

Ma J, Jones G, Zhang S, Shen J, Metzner W, Zhang L, Liang B (2003) Dietary analysis confirms that Rickett's big-footed bat (Myotis ricketti) is a piscivore. J Zool 261(3):245-248

Nagata H (1986) Female choice in Middendorff's grasshopper-warbler (Locustella ochotensis). Auk 103(4):694-700

Norberg UM, Rayner JMV (1987) Ecological morphology and flight in bats (Mammalia; Chiroptera): wing adaptations, flight performance, foraging strategy and echolocation. Philos Trans Royal Soc B 316: $335-427$

Ohdachi SD, Ishibashi Y, Iwasa MA, Fukui D, Saitoh T (2015) The wild mammals of Japan second edition. SHOUKADOH Book Sellers, Kyoto

Pastor-Beviá D, Ibáñez C, García-Mudarra JL, Juste J (2014) A molecular approach to the study of avian DNA in bat faeces. Acta Chiropterol 16(2):451-460

Popa-Lisseanu AG, Delgado-Huertas A, Forero MG, Rodríguez A, Arlettaz R, Ibáñez C (2007) Bats' conquest of a formidable foraging niche: the myriads of nocturnally migrating songbirds. PLoS One 2(2):e205

Ratnasingham S, Hebert PDN (2007) Bold: the barcode of life data system. Mol Ecol Notes 7(3):355-364. http://www.barcodinglife.org

Sorvari J, Härkönen S, Vesterinen EJ (2012) First record of an indoor pest sawtoothed grain beetle Oryzaephilus surinamensis (Coleoptera: Silvanidae) from wild outdoor wood ant nest. Entomol Fenn 23: 69-71

Vesterinen EJ, Lilley T, Laine VN, Wahlberg N (2013) Next generation sequencing of fecal DNA reveals the dietary diversity of the widespread insectivorous predator Daubenton's Bat (Myotis daubentonii) in Southwestern Finland. PLoS One 8(11):e82168

Vesterinen EJ, Ruokolainen L, Wahlberg N, Peña C, Roslin T, Laine VN, Vasko V, Sääksjärvi IE, Norrdahl K, Lilley TM (2016) What you need is what you eat? Prey selection by the bat Myotis daubentonii. Mol Ecol 25(7):1581-1594

Vesterinen EJ, Puisto AIE, Blomberg A, Lilley TM (2018) Table for five, please: dietary partitioning in boreal bats. Ecol Evol

Walker FM, Williamson CHD, Sanchez DE, Sobek CJ, Chambers CL (2016) Species from feces: order-wide identification of Chiroptera from guano and other non-invasive genetic samples. PLoS One 11(9):e0162342

Publisher's note Springer Nature remains neutral with regard to jurisdictional claims in published maps and institutional affiliations. 